\title{
A TUNABLE BIREFRINGENT FILTER FOR THE UV REGION
}

\author{
KERSTIN FREDGA* \\ Sonnenborgh Observatory, Utrecht, The Netherlands \\ and
}

J. A. HÖGBOM

Kapteyn Astronomical Laboratory, Groningen, The Netherlands

\begin{abstract}
In the near ultraviolet part of the spectrum filters with narrow bandpasses (smaller than a few Ångströms) are difficult to manufacture. Ordinary birefringent filters suffer from low transmission because of the great number of polarizers usually needed. From this point of view the Šolc-type birefringent filter offers a solution since it only employs two polarizers. With a suitable arrangement of the angles of the optic axes of the birefringent plates adjacent secondary maxima can be suppressed to any desired value.

It is shown that by modulating the distribution of the angles by a cosine function a normal transmission peak may be split into two peaks. The distance of the two peaks depends on the frequency of the modulation function. In this manner the filter can easily be tuned over half the free spectral range without appreciable change of the transmission profile. The theoretical results have been experimentally verified.
\end{abstract}

\section{The Šolc-type filter}

In the ultraviolet part of the spectrum it is difficult to manufacture optical filters with narrow bandpasses. Ordinary birefringent filters suffer from low transmission because of the great number of polarizers usually needed. From this point of view the Šlc-type birefringent filter offers a solution since it only uses two polarizers.

The Solc filter employs a pile of identical retardation plates, usually of quartz or calcite, and one polarizer at each end of the pile. The retardation plates are cut with the crystal optic axes parallel to the surface. Šolc $(1953,1959,1960,1965)$ describes two main versions of this filter. In his type I filter, called the folded filter by Evans, the axes of the plates are all rotated the same angle $\omega$ with respect to the axis of the first polarizer, but alternate between $+\omega$ and $-\omega$. The end polarizer is oriented at right angles to the first (see Figure 1). In his type II filter, called the fan filter by Evans, the angles of the retardation plates increase monotonically in the series $\omega, 3 \omega, 5 \omega$, $\ldots(2 N-1) \omega$ and with the end polarizer parallel to the first. In both filter types the angle $\omega$ is given by

$$
\omega=\pi / 4 N \quad \text { (radians) }
$$

where $N$ is the number of plates.

Evans (1958), using the Jones (1941 a, b) matrix calculus found that the on-axis transmission for both types of filter is

$$
T=A\left[\begin{array}{c}
\sin N \% \cos \gamma \sin 2 \omega \\
\sin \%
\end{array}\right]^{2}
$$

* On leave of absence from Stockholm Observatory, Saltsjöbaden, Sweden. 
the parameter $\chi$ is given by

$$
\cos \chi=\cos \gamma \cos 2 \omega \text {. }
$$

$2 \gamma$ is the retardation of a single plate at the wavelength $i$ and given by

$$
2 \gamma=2 \pi \cdot d \cdot \mu / \lambda
$$

where $d$ is the plate thickness and $\mu=\left(n_{e}-n_{0}\right)$ is the difference between the extraordinary and ordinary refractive indices. Transmission maxima occur at wavelengths for which $2 \gamma=\mathrm{k} \cdot 2 \pi$ and $2 \gamma=\left(k+\frac{1}{2}\right) \cdot 2 \pi$ for the fan filter and the folded filter respectively. The constant A depends upon the losses in polarizers and plates.

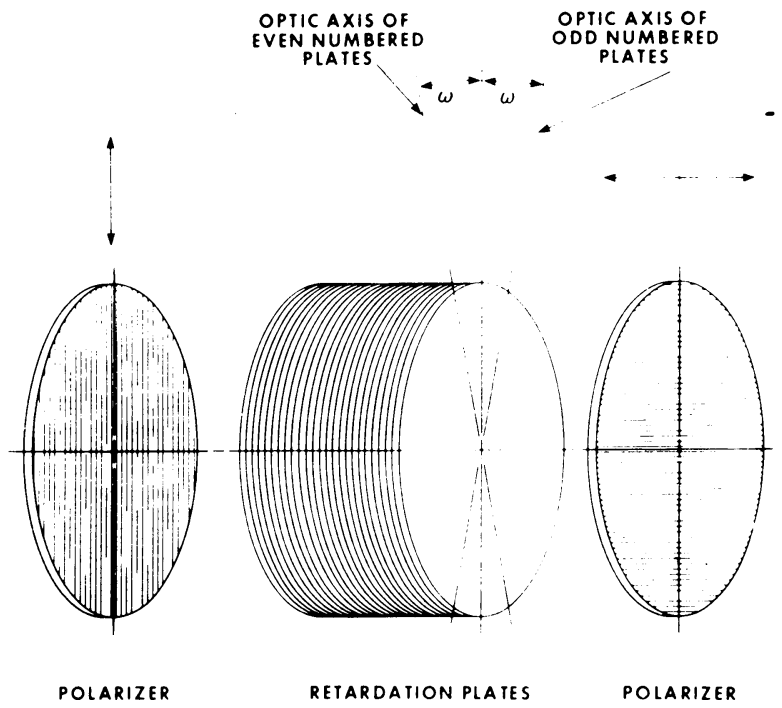

Fig. 1. Simple Solc filter of the folded type.

This filter has a bandwidth between half intensity points of

$$
\Delta \lambda_{\frac{1}{2}}=\frac{0.8 \lambda^{2}}{N \cdot d \cdot \mu} \cdot 1-(\lambda / \mu)(\partial \mu / \hat{\partial} \hat{\lambda})
$$

and sidelobes transmitting $11 \%$ of the main peak maximum.

\section{Control of Sidelobes}

Solc $(1960,1965)$ describes how the sidelobes can be suppressed by tapering the distribution of angles; the quantity $\omega$ is made larger near the centre and smaller towards the ends in a way that the more general condition

$$
\sum_{i=1}^{N}\left|\omega_{i}\right|=\frac{\pi}{4}
$$


TABLE I

Bandwidth relative to that of an untapered filter and maximum transmission in the first sidelobes for different tapering functions

$\begin{array}{llll}\text { Tapering function } & \text { Bandwidth } & \text { First sidelobe } & \text { Second } \\ \text { No tapering } & 1.00 & 11.6 \% & 3.9 \% \\ \text { Roof }(2: 1) & 1.11 & 3.4 & 2.1 \\ \text { Cosine } & 1.36 & 1.8 & 0.22 \\ \text { Triangular } & 1.47 & 0.09 & 0.47 \\ \text { Gaussian }(100: 1) & 2.00 & 0.0045 & 0.0002\end{array}$

For the profiles in Figure 2 and for most other calculations in this paper we have used a very general and flexible computer program developed by Beckers and Dunn (1965) and based on the Jones matrix calculus.

\section{Relevant Filter Parameters}

An inspection of the general form of the Jones calculus chain of matrices for any birefringent filter shows, that the physically most relevant quantities are in fact the distribution of the angle differences (between adjacent plates or between plate and polarizer) and the amplitude and relative phase of the emergent wave as function of the retardation $2 \gamma$.

As an example we investigate the two simplest types of Šolc filter i.e. those which have constant angle differences $\alpha$ throughout the filter. (The only difference between these filters and the simple fan and folded filters described earlier is that all the angle differences including those to the polarizers are the same.) The arrangement corresponding to the folded filter becomes: $0, \alpha, 0, \alpha, 0 \ldots$ and that corresponding to the fan filter: $0, \alpha, 2 \alpha, 3 \alpha, \ldots N \alpha$. The last polarizer is in both the cases oriented at right angles to the angle that an $(N+1)$ th plate would have had.

We have anlysed the transmission of these filters using Jones calculus. The on-axis transmission for both the types is

$$
T=A\left[\begin{array}{c}
\sin (N+1) \chi \\
\sin \chi
\end{array}\right.
$$

where as before

$$
\cos \%=\cos \gamma \cos \alpha
$$

a somewhat simplified expression compared to Equation (2). The transmission of the main peak is maximum when

$$
\alpha=\pi / 2(N+1)
$$

The bandwidth is determined by Equation (5) with the only change that $N$ has to be substituted by $(N+1)$ giving a slightly narrower peak with somewhat increased sidelobes. As before we can suppress the sidelobes to any desired value by tapering in 
this case, the distribution of angle differences $\alpha$. In the special case of the folded filter shown in Figure 2, the tapering of the angle differences $\alpha$ gives essentially the same plate angle distribution as tapering the angles $\omega$ themselves.

\section{Tuning the Filter}

The general shape of the transmission peaks and the changes in this shape resulting from the various types of tapering illustrated in Figure 2, suggest that the relation between the distribution of plate angles on the one hand and the transmission profile on the other is similar qualitatively to a Fourier transform. This is important, since it indicates, in a qualitative way, what changes should be done to a particular angle distribution in order to achieve some special shape of the transmission profile.

Many different shapes of the transmission profile are possible apart from the simple arrays of single peaks produced by the fan and folded filter configurations. However, if all plates have the same thickness, the profiles will always be symmetrical (on a retardation scale) about the wavelengths for which the retardation of a single plate is an integral multiple of $2 \pi$ radians of phase. One especially interesting possibility that satisfies the symmetry requirement is a double peaked profile for which the distance between the two components may be chosen at will. The individual maxima could in this case be tuned in wavelength by simply changing the distribution of plate angles.

If we had been dealing with a true Fourier transform relation, we could have produced such a double peak by multiplying the original function, i.e. the distribution of angle differences, by a cosine function of a certain periodicity; the frequency $s$ (cycles per unit length) of the cosine function would determine the distance between the two peaks. It turns out that, although the actual relation is not a true Fourier transform, this particular rule about cosine modulation can be used also in this case, subject to certain restrictions that will not be discussed here.

Figure 3 shows the effects on the filter profile of a cosine modulation in the angle difference distribution for various values of the modulation frequency $s$ (here expressed in terms of cycles per plate). As $s$ goes from 0 to 0.5 the filter changes from the fan type, via split peak versions, into the folded type. When $s=1,2,3$ etc. we have modulated an integral number of full cycles over the distance from one plate to the next and returned to the original plate angle distribution.

The theoretical conclusions discussed above have been tested experimentally using a set of 16 individually mounted quartz plates. The experimentally determined transmission values fit the theoretical values within $1 \%$ of the transmission of the main peak. This means that at the scales used in Figures 2 and 3 the difference between experimental and theoretical curves would hardly be noticable.

\section{Applications}

As we have seen above it is possible with a set of identical retardation plates which can be rotated independently to choose a suitable transmission profile and to tune 

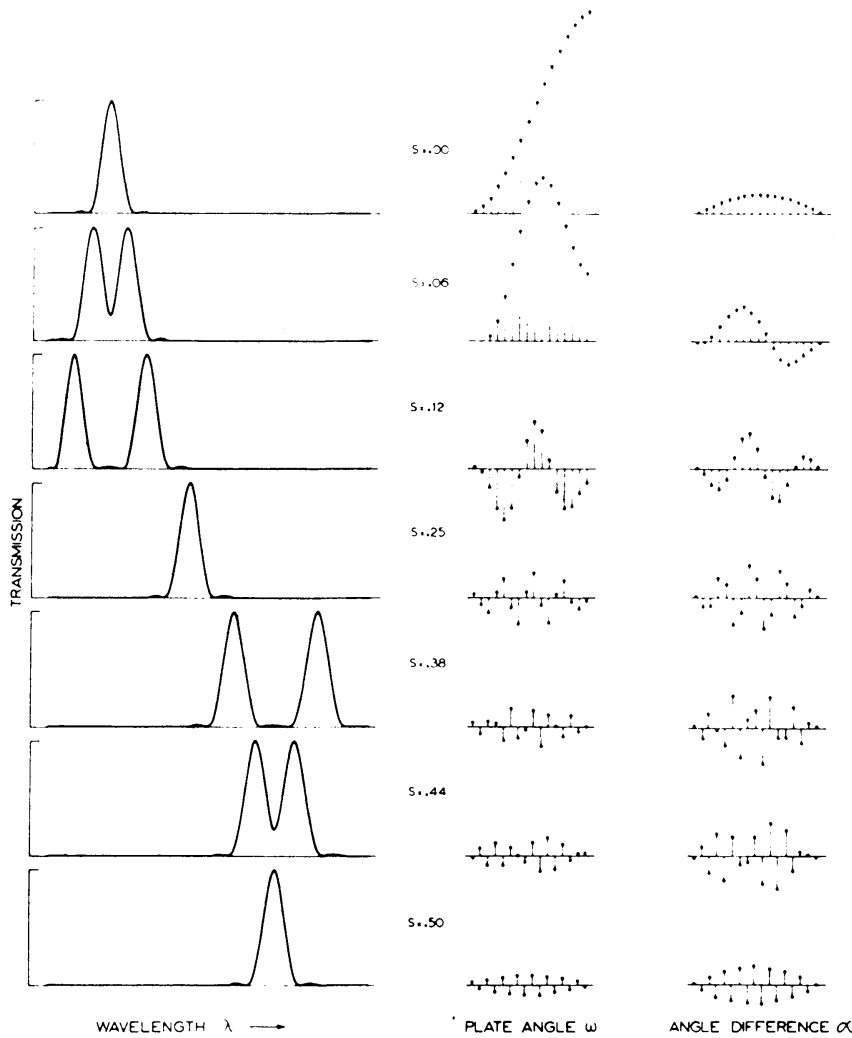

Fig. 3. Splitting of peaks and tuning by cosine modulation. Represented are from left to right, transmission profiles, plate angles $(i)$ and angle differences $x$ for different frequencies of the modulation function. By modulation through half a cycle ( $s$ goes from 0.0 to 0.5 ) the filter has transformed from a fan filter into a folded filter. This example is based on a cosine tapered filter with 16 plates.

this in wavelength without changing the maximum transmission or the shape of the profile.

To illustrate the previous discussion we have calculated a filter for observing the $\mathrm{H}$ and $\mathrm{K}$ lines of $\mathrm{Mg}$ II situated at $2802.7 \AA$ and $2795.5 \AA$. We have choosen a cosine tapered filter consisting of 50 quartz plates with a total thickness of birefringent material of $29 \mathrm{~mm}$. The bandpass of such a filter is $2 \AA$ in the $2800 \AA$ region, with the first sidelobe transmitting only $1.8 \%$. The next reoccurring peak in the spectrum appears about $100 \AA$ away.

A filter with such specifications is quite feasible. The described example is similar to filters flown in Aerobee rockets by Fredga (1969a, b).

If we want to reach the $\mathrm{H}$ or the $\mathrm{K}$ line by tuning we design the filter with the plate thickness such that the ordinary single peak is at a distance of about $25 \AA$ away, (i.e. $\frac{1}{4}$ of the distance to the reoccurring peak). Then by a cosine modulation with, in this particular example, $s=0.21$ we reach the $\mathrm{K}$ line and with $s=0.29$ we reach the $\mathrm{H}$ line. 
If we want to record the combined emission of the $\mathrm{H}$ and the $\mathrm{K}$ lines we can do this with the same filter performing two modulations after each other. We first split the ordinary peak in two peaks separated by a distance corresponding to the distance between the $\mathrm{H}$ and the $\mathrm{K}$ lines. Then by modulating a second time this double peak as a unit may be tuned to the position where the two peaks coincide with the $\mathrm{H}$ and the $\mathrm{K}$ lines respectively. A 'square' filter profile can be produced with a more complex distribution of angle differences. These examples are illustrated in Figure 4.

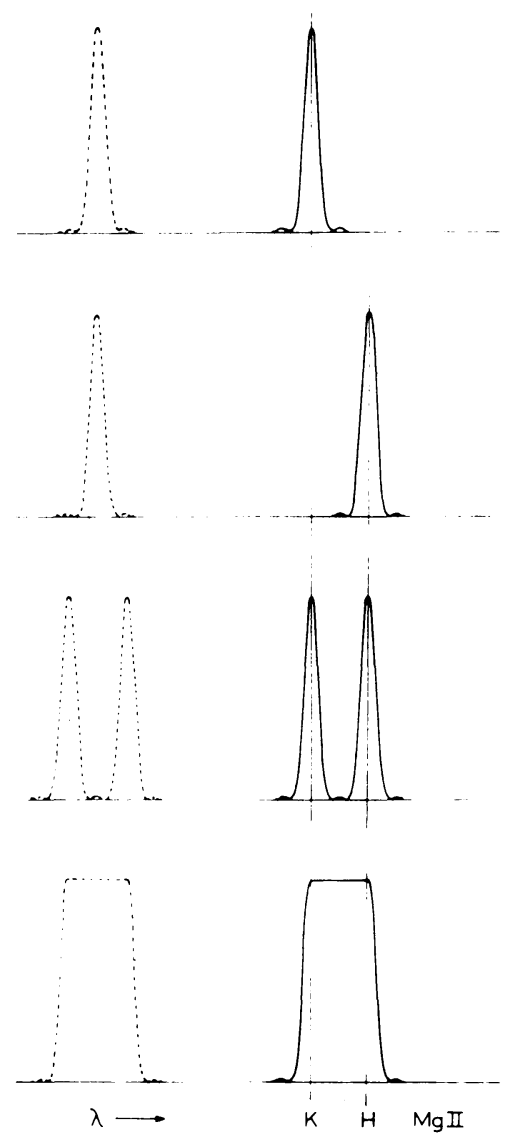

Fig. 4. Example of possible filter profiles obtainable with the same set of retardation plates for observing the $\mathrm{H}$ and $\mathrm{K}$ lines of $\mathrm{MgII}_{\mathrm{I}}$ at $2800 \AA$. Example based on a cosine tapered filter with 50 plates (see text).

The alignment of the crystal optic axes of the plates (i.e. the angle $\omega$ ) is not very critical. Errors as large as $0.5^{\circ}$ are negligible. This accuracy is easily obtained.

The main difficulty in manufacturing Šolc-type filters is in cutting plates of equal thickness. It is desirable to control the optical thickness of a single plate to \pm 0.01 orders which takes great manufacturing skills. The plates used in our experiments have been cut by Šlccs group in Czechoslovakia and obviously meet very high demands. 
The possibility of tuning may facilitate the manufacturing of the filters in the sense that the absolute thickness of the plates are not so critical as long as they are equally thick. A more comprehensive report on this investigation is in preparation (Fredga and Högbom, 1971).

\section{References}

Beckers, J. M. and Dunn, R. B.: 1965, Air Force Camb. Res. Lab. Instrumentation papers No. 75. Evans, J. W.: 1958, J. Opt. Soc. Am. 48, 142.

Fredga, K.: 1969a, Appl. Opt. 8, 333.

Fredga, K.: 1969b, Solar Phys. 9, 358.

Fredga, K. and Högbom, J. A.: 1971, Solar Phys. (in press).

Jones, R. C.: 1941a, J. Opt. Soc. Am. 31, 488.

Jones, R. C.: 1941b, J. Opt. Soc. Am. 31, 500.

Šolc, I.: 1953, Česk. Časopis Fys. 3, 366.

Šolc, I.: 1959, Czech. J. Phys. 9, 237.

Šolc, I.: 1960, Česk. Časopis Fys. 10, 16.

Šolc, I.: 1965, J. Opt. Soc. Am. 55, 621. 